\title{
¿Hemos cambiado? La perspectiva de los profesores eméritos sobre el cambio educativo en la universidad
}

\section{Have we changed? Professors emeriti's views on educational change at university}

\author{
Felipe Trillo Alonso ${ }^{1 *}$ \\ felipe.trillo@usc.es \\ Miguel Zabalza Beraza* \\ miguel.zabalza@usc.es \\ María Ainoa Zabalza Cerdeiriña** \\ mzabalza@uvigo.es \\ * Universidad de Santiago de Compostela, España \\ ** Universidad de Vigo, España
}

\section{Resumen:}

El presente trabajo forma parte de una investigación más amplia desarrollada en el marco de una convocatoria nacional de $\mathrm{I}+\mathrm{D}^{2}$ en la que han participado varias universidades españolas. Su objetivo principal fue recuperar el conocimiento experto del profesorado emérito, esto es, reconstruir el proceso personal y profesional seguido por profesores-as que han sido muy relevantes en el mundo de la docencia y de la investigación.

En este trabajo se presentan e interpre$\tan$ las reflexiones que sobre los procesos de cambio, de reforma, de innovación y/o

\begin{abstract}
:
The present study is part of a broader investigation developed within the framework of a national $R$ \& $D$ project in which several Spanish universities participated. Its main objective was to recover the expert knowledge of professors emeriti, that is to reconstruct the personal and professional processes followed by professors who have been very relevant in the world of teaching and research.

This study presents and interprets the answers expressed by 20 professors emeriti from 13 Spanish public universities regarding the processes of change, reform, in-
\end{abstract}

1 Dirección para correspondencia (correspondence address):

Felipe Trillo Alonso. Dpto. de Pedagogía y Didáctica. Universidad de Santiago de Compostela. Facultad de Ciencias de la Educación. Campus Vida.15782 Santiago de Compostela (España).

2 Título de Proyecto: Recuperación y representación del conocimiento experto del profesorado emérito de las universidades españolas: ingeniería del conocimiento para la reconstrucción de la misión de la universidad y la calidad de la docencia universitaria en el marco del proceso de convergencia europeo. Financiación del Ministerio de Economía y Competitividad. 
¿Hemos cambiado? La perspectiva de los profesores eméritos sobre el cambio educativo en la universidad

Felipe Trillo Alonso, Miguel Zabalza Beraza y María Ainoa Zabalza Cerdeiriña

de mejora educativa en la universidad, expresan 20 profesores eméritos de 13 universidades públicas españolas. El acceso a la visión personal de los profesores eméritos se efectuó mediante la realización de una entrevista semi-estructurada. Los datos que resultan de sus respuestas han sido sometidos a un análisis de contenido del que se derivan muchas consideraciones, valoraciones y recomendaciones dignas de ser tomadas en consideración para la comprensión de las transformaciones acaecidas en la universidad a lo largo del amplio período que abarca su trayectoria profesional (1965-2015).

\section{Palabras Clave:}

Enseñanza universitaria; elicitación del conocimiento experto; profesorado emérito; cambio e innovación en la universidad. novation and/or educational improvement which Spanish universities have undergone. Using semi-structured interviews, we try to access to the personal vision of professors emeriti. Their answers to the questions that we have raised were analyzed through content analysis. Many considerations, insights and recommendations, worthy of being taken into consideration, derive from this analysis. We will refer, in particular, to their beliefs and insights regarding the transformations that took place in Spanish universities throughout the period that covers the professional career of these professors emeriti (1965-2015).

\section{Keywords:}

University education; elicitation of expert knowledge; professor Emeriti; change and innovation in the university.

\section{Résumé:}

Ce travail fait partie d'une recherche plus large développée dans le cadre d'un concours en recherche et développement à l'échelle nationale auquel ont participé plusieurs universités espagnoles. Son principal objectif était de recueillir l'expertise de professeurs émérites, c'est-à-dire de reconstituer les processus personnels et professionnels qui ont été suivis par ces derniers et qui ont particulièrement fait avancer le monde de I'enseignement et de la recherche.

Dans cet article, les réponses exprimées par 20 professeurs émérites de 13 universités publiques espagnoles sur les processus de changement, de réforme, d'innovation et/ou d'amélioration de l'éducation dans I'université sont présentées et interprétées. En utilisant des entretiens semi-directifs, nous essayons d'accéder à la vision personnelle des enseignants émérites. Leurs réponses aux questions que nous avons soulevées ont été analysées via une analyse de contenu. De nombreuses considérations, évaluations et recommandations qui découlent de cette analyse sont dignes d'être prises en compte, surtout pour comprendre les transformations qui ont eu lieu dans l'université tout au long de la période qui couvre leurs carrières professionnelles (1965-2015).

\section{Mots clés:}

Enseignement universitaire; élicitation de connaissances expertes; professeurs émérites; changement et innovation à l'université.

Fecha de recepción: 7-2-2018

Fecha de aceptación: 2-5-2018 
Felipe Trillo Alonso, Miguel Zabalza Beraza y María Ainoa Zabalza Cerdeiriña

\section{Introducción}

Contribuir al conocimiento y comprensión del cambio educativo en la Universidad es el objetivo de este trabajo. La originalidad de nuestra aportación radica en tomar como "informantes clave" de nuestro objeto de estudio a un colectivo singular: el de los profesores eméritos (entre los que contamos con un premiado con el Príncipe de Asturias); profesores, en fin, que han accedido a esa categoría como consecuencia de una dilatada y fructífera experiencia académica al servicio de las universidades en las que trabajaron. Nuestro trabajo forma parte de un proyecto de investigación más amplio, sobre recuperación del conocimiento experto. Proyecto ya finalizado, del que nos gusta pensar que, como mínimo, ha servido para preservar el singular bagaje experiencial de quienes han sido profesores y profesoras universitarias de relevancia en la Universidad española durante los últimos cincuenta años.

Convencidos como estamos de que "las personas que han adquirido la categoría de eméritos han acumulado una experiencia académica inestimable", sostenemos que conocer sus percepciones sobre el cambio educativo experimentado por la universidad española en los últimos decenios, representa una perspectiva sumamente interesante que puede contribuir a la mejora de la calidad universitaria; al menos en cuanto se refiere a su definición y orientación. Considérese, en este sentido, que algunas de las biografías docentes de nuestros informantes se inician ya en el año 1962 (fueron estudiantes en la década de los cincuenta del siglo pasado). La sola mención de esas fechas, da idea de una larga trayectoria en la que tanto el contexto social y político, como la propia universidad española han pasado por transformaciones muy relevantes. No solo la transición del régimen dictatorial al sistema democrático, sino la aprobación de la LRU, los sucesivos cambios en los planes de estudios, la aparición de las TIC, el proceso de articulación del Espacio Europeo de Educación Superior (EEES) y, ya en el marco de la LOU, el nuevo encaje social de las universidades, la tensión entre la docencia y la investigación en un marco en el que se prima la transferencia de conocimiento como fuente de financiación, los procesos de acreditación de titulaciones y profesorado a través de agencias externas de evaluación, el proceso de des-funcionarización de los docentes, etc.

Conscientes, pues, de lo relevante que resulta conocer este tiempo histórico contemporáneo para comprender lo que acontece en la ac- 
tualidad, básicamente lo que hacemos en este trabajo es recuperar el "conocimiento experto" (no de especialistas, sí de experimentados) de profesores y profesoras que no sólo han vivido, sino que incluso han marcado una época en el desarrollo de nuestras instituciones universitarias. Lo cual, en esencia, implica como se decía en el proyecto: "reconstruir el proceso personal y profesional seguido por personas que han sido muy relevantes en el mundo de la docencia y de la ciencia; recuperar las convicciones sobre su trabajo que han ido generando a lo largo de su carrera profesional; recuperar su narrativa sobre las prácticas docentes que han realizado y cómo éstas han ido evolucionando a lo largo del tiempo; recuperar sus discursos sobre la universidad y su función social".

Siguiendo esa directriz general, el objetivo más concreto de este trabajo consiste en analizar lo que los profesores eméritos dicen específicamente sobre el cambio educativo. Analizando y sistematizando sus respuestas, hemos elaborado un marco de referencia que nos permita repensar, diferenciándolos, los procesos de cambio, de reforma y de innovación y mejora educativa en la universidad.

\section{Marco de investigación}

Este trabajo sobre el cambio educativo en la Universidad participa del mismo marco de referencia teórico y metodológico del proyecto de investigación, ya citado, del que forma parte. Siendo así, este trabajo se inserta en una amplia tradición investigadora sobre la recuperación del conocimiento experto de los profesores que presenta como principales referentes los siguientes: (a) las investigaciones sobre el pensamiento de los profesores ("teacher thinking movement"), especialmente referidas al ámbito universitario (Fox, 1983; Gow y Kember, 1993; Samuelowicz, 1999; Samuelowicz y Bain, 2001; Hativa \& Goodyear, 2002); (b) las investigaciones sobre "buenas prácticas docentes" (Bain, 2006; Koppelman, 2005; Feixas, 2002); (c) las investigaciones sobre "creencias asumidas" ("espoused beliefs": Finkel, 2008; Kember, 2001); (d) las investigaciones sobre "historias de vida" (Bolívar, 2006; Moreno, 2002); (e) las investigaciones sobre la construcción de la "identidad profesional" (Barnett y Di Napoli, 2007; Hankel, 2000); (f) las investigaciones sobre innovación y cambio en Educación Superior (Barnett, 1992, 1997; Stanley and Trinkle, 2011; Swanger, 2016). 
En cualquier caso, el eje de nuestros análisis es el cambio institucional. No se nos oculta la extraordinaria complejidad de las cuestiones vinculadas a la innovación y cambio en las universidades. Como ha señalado Fullan (2002) se trata de procesos condicionados desde múltiples frentes: condiciones estructurales (sociales y políticas; ideológicas), condiciones institucionales (organizativas, de participación, de recursos), y condiciones personales (intelectuales, morales e incluso emocionales de las que depende cualquier posibilidad de colaboración). Cambio, reforma, innovación, mejora, son conceptos que se refieren a temas de gran resonancia social, que pueden ser sencillos en su definición pero que son, como dijimos, extremadamente complejos en su desarrollo, fundamentalmente porque durante su realización son susceptibles de interpretaciones diversas y hasta contrapuestas y, por ello, potencialmente generadoras de conflictos (Escudero, 1999; Fullan, 2002).

Aún así, o precisamente por ello, resulta imprescindible disponer de una conceptualización que nos permita precisar lo siguiente: primero, que hablamos de "cambio educativo" cuando nos referimos a algún tipo de modificación -planificada o no- de la realidad educativa, que puede observarse comparando simplemente la situación de inicio con la de finalización del proceso (Guarro, 2005); un cambio, en todo caso, que puede ser para bien, pero también para peor (Escudero, 2002). Segundo, que hablamos de "reforma educativa", cuando nos referimos a iniciativas diseñadas y dirigidas por instancias políticas (gobiernos, organismos internacionales), generalmente planteadas a nivel macro, habitualmente con formato y rango de Ley, y que se presentan, por lo general, con carácter prescriptivo (Bolívar, 2002; Bolívar y Rodríguez, 2002; Gairin, 2010; Rivas, 2000). Tercero, que hablamos de innovación educativa, cuando nos referimos a cambios que afectan a las dimensiones más cualitativas de la educación, lo que implica un cambio profundo en las concepciones de los directamente involucrados (Díaz Barriga, 2010). Supone la innovación una práctica deliberada y sistemática, a nivel meso y micro, configurada por tres dimensiones básicas que se exigen mutuamente: la alteración voluntaria de creencias y supuestos previos, la adopción reflexiva de nuevos enfoques y la utilización comprensiva de nuevos materiales o recursos (Fullan, 1982; Escudero y González, 1987; Zabalza y Zabalza Cerdeiriña, 2012). Según esto, la innovación es acción, pero es acción justificada no mero activismo; por lo mismo, no cabe confundirla con una adopción oportunista y burocrática de ciertos usos y maneras, 
algo meramente aparente. Cuarto, que hablar de "mejora educativa" nos sitúa en un ámbito más valorativo que descriptivo; sujeto, por lo tanto, a criterios de calidad educativa que sería preciso definir previamente; no obstante, existe una suerte de consenso tácito que nos lleva a pensar que, a diferencia de los cambios, que pueden ser para bien o no, toda innovación, que realmente lo sea (según explicamos), sería sinónima de mejora.

\section{Objetivos}

El objetivo general de nuestro texto, en consonancia con el que fue objetivo general de todo el proyecto, es el de recuperar el conocimiento y la experiencia del profesorado emérito de forma que pueda servirnos de punto de referencia en el permanente debate sobre el cambio y la innovación educativa en la universidad.

En lo que se refiere a este trabajo, ese objetivo general se concreta en 3 objetivos específicos: (a) recuperar los elementos más relevantes del conocimiento y experiencia personal de 20 profesores eméritos en torno a la innovación y cambio en la universidad; (b) Analizar y visibilizar sus comentarios y valoraciones de forma que puedan ser tomadas en consideración en sede universitaria y (c) Contrastar las ideas aparecidas entre sí y con las que han servido a los autores de este texto como marco conceptual de referencia.

\section{Metodología}

Los estudios sobre el pensamiento de los profesores y las historias de vida, así como las modalidades de acceso y análisis del conocimiento experto, son los referentes metodológicos de este trabajo. Desde esa perspectiva, se trata de acceder a la visión personal de profesionales de reconocido prestigio contada por ellos mismos. En esa dirección, nos valemos de la entrevista semiestructurada ${ }^{3}$ como recurso técnico. En ella se abordaron muchos y diferentes aspectos relacionados con la biografía profesional de los profesores: "cómo ha sido su vida docente, cómo han construido su idea de universidad y su identidad como profesores

3 El guión completo se puede consultar en el enlace: http://www.scielo.org.mx/pdf/redie/v18n1/v18n1a13.pdf 
y profesoras universitarios, qué piensan de la universidad y su función (y cómo ha ido evolucionando esa idea desde que comenzaron su vida universitaria), cómo plantearon los procesos de enseñanza-aprendizaje en las disciplinas en las que actuaron como docentes, cómo vincularon su actividad docente con la investigadora, cómo han visto evolucionar a sus estudiantes, qué opinan de los actuales planteamientos de la docencia universitaria". Fueron en total 26 preguntas cuyas respuestas se grabaron en audio y se transcribieron. Su análisis se llevó a cabo a través del sistema de análisis de contenido explicitado en el modelo de categorización "constant comparison method" (Glaser y Strauss, 1967; Dye, Schartz, Rosenberg y Coleman, 2000) para identificar los "patrones de respuesta". La identificación de dichos patrones representa, según Koppelman (2005), un intento de capturar la esencia del conocimiento y las prácticas de sujetos relevantes (knowledge capturing, Becerra-Fernández y Sabherwal, 2010) para expresarla de una manera concisa de forma que se facilite su comunicación.

Del conjunto de ideas identificables en las entrevistas con los/las eméritos hemos centrado nuestras consideraciones en sus aportaciones en torno al cambio educativo: cómo han vivido y valoran los cambios producidos en la universidad desde los años 50 del pasado siglo hasta nuestros días ${ }^{4}$.

\section{Muestra}

En este artículo se abordan las aportaciones de $20^{5}$ profesores y profesoras eméritos: 17 hombres y 3 mujeres, todos españoles, con edades comprendidas entre los 62 y los 83 años, y con un tiempo de dedicación a la docencia que va de los 25 a los 53 años.

Los profesores cuyas ideas analizamos desarrollaron su docencia e investigación en 13 universidades españolas: Cantabria (1), Complutense (2), La Laguna (1), Las Palmas de Gran Canaria (1), Oviedo (1), País Vasco(1), Politécnica Madrid (1), Pública de Navarra (1), Salamanca (1), Santiago de Compostela (5), Vigo(4), Zaragoza (1).

4 En este artículo, nos centramos sólo en 13 preguntas del total de 26; pues seleccionamos sólo las más y mejor relacionadas con nuestro objeto de estudio.

5 En el proyecto general participaron investigadores de 7 universidades y fueron entrevistados 155 profesores y profesoras eméritos (140 españoles y 15 extranjeros). 
¿Hemos cambiado? La perspectiva de los profesores eméritos sobre el cambio educativo en la universidad

Felipe Trillo Alonso, Miguel Zabalza Beraza y María Ainoa Zabalza Cerdeiriña

Asimismo, se reparten de este modo por grandes áreas de conocimiento y titulaciones. Ciencias Tecnológicas: Ingeniería de Minas (IdM1); Ciencias Experimentales: Física (F)-1; Química (Q)-4; Ciencias de la Salud: Medicina(M)-2; Farmacia (Fa)-1; Psicología (Ps)-1; Ciencias Sociales y Jurídicas: Derecho (D)-1; Comunicación (Periodismo) (C)-1; Economía (E)-2; Pedagogía (P)-1; y Ciencias Humanas: Geografía e Historia (G)-1; Filología (Fi)-1; Filosofía (Fía)-1; Teología (T)-1; Bellas Artes (BA) $-1^{6}$.

\section{Análisis e interpretación de la información}

El análisis de los datos tiene una orientación básicamente cualitativa. Como en el proyecto general, el análisis se realiza a tres niveles: individual (intra-sujeto); como grupo de docentes (inter-sujetos) identificando planteamientos e inquietudes comunes al conjunto de la muestra; y por grupo de especialidad, tratando de detectar (pero sin hipótesis previa) la existencia de alguna tendencia común por grandes áreas de conocimiento científico. No obstante, lo que presentamos aquí es sólo el análisis inter-sujetos, por razones de espacio y por ser el que ofrece una información más relevante en este caso.

Centrándonos pues, en el análisis inter-sujetos, pasamos a analizar las respuestas que estos docentes dan a las preguntas relativas a los procesos de cambio en la Universidad. En esa dirección, utilizando el análisis de contenido explicitado en el modelo de categorización propuesto por Glaser y Strauss (1967) que ya antes mencionamos, y una vez establecidas las categorías por el marco teórico, la adscripción de las reflexiones de los entrevistados a las mismas se hizo mediante un "acuerdo interjue-

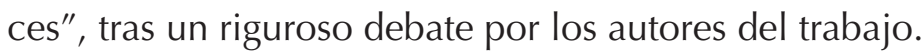

Por supuesto, no transcribimos todo lo que dicen sino solo las apreciaciones más elocuentes a algunos de los tópicos. Por razones de espacio hemos dejado fuera de consideración otras cuestiones que será preciso retomar en otro momento (por ejemplo, valoraciones sobre la evolución de los departamentos que encerró a los profesores en sus áreas de conocimiento y dificultó la interdisciplinariedad; sobre la incorporación de pleno derecho de la mujer; sobre la apertura de la universidad a

6 Las abreviaturas señaladas servirán para identificar a los informantes en el siguiente apartado; cuando hay más de uno en una titulación se numeran consecutivamente. 
amplios estratos de la población; sobre las relaciones entre profesores y estudiantes; sobre el impacto de las TIC; sobre la internacionalización; sobre los extraordinarios avances en recursos materiales para la investigación en las áreas de ciencias experimentales e ingenierías; sobre la mejor cualificación de los profesores jóvenes en sus respectivos campos de investigación, en contraste con los escasos avances en la formación docente inicial de esos mismos profesores; etc.).

Tomadas en su conjunto, las aportaciones de los 20 eméritos con los que hemos trabajado se podrían sintetizar en un marco conceptual en el cual el análisis de la universidad se proyecta en torno a 4 grandes espacios temáticos: la propia universidad y sus dinámicas internas; la sociedad a la que sirve y sus demandas; el profesorado cuyos miembros constituyen sus agentes primarios; la propia innovación como esfuerzo colectivo por la calidad. A continuación exponemos los temas extraídos de las entrevistas incluyendo en cada uno de ellos las opiniones y valoraciones de los entrevistados y nuestros propios comentarios al respecto.

\section{La universidad como tal en el foco de análisis}

Aunque el orden en que analizaremos las aportaciones de los eméritos no tiene que ver ni con la importancia ni con la frecuencia en que aparecieron en las entrevistas, parece conveniente iniciar el recorrido por las referencias que se hacen a la universidad.

\subsection{ReDEFINICIÓN DE LAS SEÑAS DE IDENTIDAD DE LA UNIVERSIDAD}

No hay duda de que repensar la razón de ser de la universidad, la visión y misión que los planes estratégicos institucionales recogen, las perspectivas y finalidades que los diversos colectivos le atribuyen es una cuestión fundamental. La forma en que definimos la identidad de la universidad acaba configurando nuestra propia identidad como docentes. Por eso es tan importante la respuesta que dan estos profesores eméritos a la pregunta qué es la universidad y para qué sirve.

Bastantes de nuestros eméritos personalizan la universidad identificándose con ella. No es una institución ajena que les acoge, ese lugar en donde trabajan, sino que ellos son la universidad, que es algo muy distinto a decir que la universidad son ellos. Tiene que ver con el sentido de pertenencia frente al desapego, ese algo que lleva aparejada la corresponsabilidad frente a la indiferencia. Así, el devenir histórico, la 
¿Hemos cambiado? La perspectiva de los profesores eméritos sobre el cambio educativo en la universidad

Felipe Trillo Alonso, Miguel Zabalza Beraza y María Ainoa Zabalza Cerdeiriña

realidad actual, las posibilidades de futuro de la universidad no son algo que se describe, sino algo que señalan como recorrido vivido en primera persona. Por eso las preguntas iniciales de qué es la universidad y para qué sirve se traducen para nuestros entrevistados en qué somos y para qué servimos como universitarios. Como colectivo, no como profesores aislados y tomados de uno en uno. En este sentido, sus reflexiones son en realidad un atreverse a cuestionar muchos supuestos vitales y no sólo institucionales. He aquí sus palabras:

1- La Universidad debe avanzar hacia la conciencia de su propia dignidad. La universidad fue y tiene que seguir siendo lugar de cultivo de la Ciencia, del diálogo, de la Cultura, de la Justicia. (C-1)

2- Me disgusta que como consecuencia de los nuevos planes la universidad se entienda como algo orientado a obtener alguna ventaja pecuniaria ... la universidad se hizo para otras cosas (E-2)

3- La Universidad no puede ser una fábrica de prácticos destinados a obtener trabajo en una empresa. Entre otras cosas, porque la Universidad no puede, no debe estar al servicio de la empresa. Su misión es otra: la ciencia- que también interesa a la empresa, aunque ordinariamente más a largo plazo-, el enriquecimiento cultural, la búsqueda de la verdad, el fomento de las virtudes de la convivencia ciudadana ... Todo esto, hoy a algunos -incluso profesores- les suena a romanticismo. Si la Universidad abdica de su tradicional misión, sobra. (C-1)

4- Esta es otra obsesión mía, la de que la universidad se está polarizando mucho cara al mercado ... sobe todo las facultades experimentales ... la industria, en cierta manera, le está imponiendo a la universidad ciertos requisitos para que los alumnos tengan determinadas condiciones y, por supuesto, también está condicionando la investigación ... yo añoro esa universidad, si quieres romántica, donde el valor del conocimiento per se es realmente nuestra joya de la corona y, por supuesto, nadie niega que haya que ser práctico y realista y transformar ese conocimiento en hechos útiles para la sociedad. (M-1)

5- La universidad debe ser un centro independiente de pensamiento. Hoy en día, sobre todo en ciertos ámbitos, como el de la economía y, también, en otras ciencias... el pensamiento está atado a intereses. Te pongo el caso del calentamiento global. Hay investigadores en el mundo que están siendo financiados por grupos de interés como los petroleros para demostrarnos que es cierto que no hay calentamiento. Entonces, el papel de las universidades es tratar estos temas con independencia de criterios. (E-1) 
Felipe Trillo Alonso, Miguel Zabalza Beraza y María Ainoa Zabalza Cerdeiriña

6- La universidad parece no satisfacer en la calidad necesaria a sus alumnos. Éstos demandan cualificaciones profesionales y la universidad les suministra sobreformación, encerrándose en la función de crear y transmitir cultura. La solución a este dilema se resolverá diversificando la educación debido a la demanda de nuevos conocimientos, los cuales van a responder a las exigencias de la economía de mercado. (Q-2)

7- Siempre me pareció importante la vinculación de la investigación a las necesidades y temáticas que surgen del ámbito profesional (la dirección de centros, la orientación). (P-1)

Si consideramos las dos primeras manifestaciones, lo primero que Ilama la atención es la presunción de una misión canónica de la universidad como institución consagrada al conocimiento; atenta, sí, a la realidad, pero para transcenderla mediante el análisis crítico con independencia de asumir o no el liderazgo de su transformación. Hay en todo eso un halo de espiritualidad que se enraíza en la idea de la universidad como "Alma Mater", y que evoca claustros y campus habitados por una aristocracia intelectual, cuya actividad, siempre altruista, es valiosa en sí misma independientemente de su proyección social más inmediata, pero sin menoscabo de ésta; según lo cual, correspondería a otros el aprovechamiento útil del conocimiento.

Sostener que una visión así ha periclitado por completo, supone aceptar que ha habido un importante cambio respecto al sentido de la universidad. ¿Cambio a peor? No todos lo valorarían así (por ejemplo, la penúltima de las manifestaciones recogidas). Lo cierto es que estamos ante un tema que suscita un importante debate en el seno de la comunidad universitaria, por más que son muchos los que se esfuerzan por silenciarlo.

Las manifestaciones tercera, cuarta y quinta no reivindican el saber por el saber sino la independencia de éste respecto a los intereses coyunturales (para algunos espurios) del Mercado. La idea es que la universidad tiene un compromiso con el conocimiento, que debe anteponerse incluso a las exigencias inmediatas de los que pueden llegar a ser sus patrocinadores (ya sea la empresa privada o la administración pública). Según eso, estaríamos acudiendo a un discurso moral que se reclama de una ética para hablar de la universidad: ¿Cuál ética? ¿Cómo se entiende? ¿Quiénes la comparten? Son preguntas que en la institución se están evitando, forzada por buscar soluciones urgentes y pragmáticas a los problemas cotidianos, entre los que destaca la financiación. 
La penúltima de las manifestaciones recogidas es una voz discrepante. Sólo una, pero no podemos ignorar que ése es un pensamiento casi hegemónico hoy en día.

Por último, creemos que conviene tender puentes, tal como sugiere, a nuestro entender, la última manifestación recogida. En efecto, hay que estar atento a las necesidades emanadas de la realidad profesional. Ahora bien, acudimos a la interpretación que resulta del análisis intra-sujeto para entender correctamente lo que el profesor entrevistado quería decir: él defiende como algo previo el contraste con el conocimiento científico de todas las demandas sociales planteadas, pues no todas las necesidades sentidas ni todas las versiones oficiales al respecto son capaces de resistir un análisis hecho con rigor e independencia de criterio.

Subyace a las ideas expuestas, como no podía ser menos, la condición de autonomía universitaria. Pero reclamarla no exime a la universidad de ser eficaz en el cumplimiento de su función social y transparente en cuanto a sus decisiones. Autonomía comprometida, en primer lugar, en la definición de la propia identidad que en modo alguno ha de quedar al albur del dictado ajeno. Definición que debería ir más allá de las declaraciones documentales oficiales tipo Planes Estratégicos y Planes de Responsabilidad Social -que muy pocos conocen, menos aún leen, y, todavía menos, tienen en cuenta en sus realizaciones cotidianas-. Definición de la propia identidad que no se substancie en el mero plegarse a las exigencias del mercado ignorando consideraciones éticas que exigen posicionamientos críticos ante las demandas sociales.

\section{Interacción universidad-sociedad}

Aunque el sentido de las preguntas aludía de forma directa sobre los procesos de cambio en la universidad, los eméritos se han referido, también, al importante papel que la universidad juega (debiera jugar) con respecto a la sociedad. Sus aportaciones podríamos ubicarlas en dos dimensiones: la función social de la universidad y las políticas sociales.

\subsection{LA FUNCIÓN SOCIAL DE LA UNIVERSIDAD}

La sensación que producen los textos de las entrevistas analizadas es que para buena parte de los eméritos estudiados, la universidad asume importantes compromisos con la sociedad a la que pertenece. 
¿Hemos cambiado? La perspectiva de los profesores eméritos sobre el cambio educativo en la universidad

Felipe Trillo Alonso, Miguel Zabalza Beraza y María Ainoa Zabalza Cerdeiriña

1- Debería plantearse también una revisión de la función social ... en vez de centrarse en las titulaciones y las investigaciones, en vez de estar pensando siempre en el desarrollo de ella misma, darse cuenta de que ella [la universidad] es un instrumento dentro de un marco social. (P-1)

2- La universidad debería caminar hacia un mayor compromiso crítico con la sociedad... [en vez de eso] es una universidad que se mira el ombligo ... En la época en la que éramos estudiantes la universidad estaba presente para aceptar, para criticar, para modificar la realidad, y nos daban hostias por todas partes. (Psi-1)

3- El progreso científico, la aplicación de los conocimientos, el uso de la tecnología en la vida, por supuesto es muy importante, pero no sólo eso, también la política. El compromiso de la Universidad con los valores ciudadanos y sociales. Ver si una cosa tiene alguna deriva negativa para la sociedad, también. El comprometerse. Por ejemplo, problemas de emigración, inmigración, problemas de cuestiones de conflictos sociales. La Universidad no puede ser algo aislado, no puede permanecer ajena, forma parte de la sociedad y si hablamos de la Universidad actual, no está abierta. (IdM-1)

4- Manifestamos que la nueva planificación de un sistema universitario tiene que hacerse con criterios exclusivamente académicos. La universidad, por todo eso, debe ser independiente del poder político en sus dos aspectos fundamentales: transmisión de conocimientos y creatividad. ... [Ahora bien] ... el pleno ejercicio de la autonomía implica la capacidad de asumir las nuevas responsabilidades sociales con criterios de eficacia y transparencia. (Q-2)

Estas manifestaciones aluden a la función social de la universidad desde una mirada alejada de la contemporización cuando no complicidad en el mantenimiento de un statu quo establecido por diferentes instancias de poder. Una universidad comprometida en preservar su libertad de pensamiento para actuar como contrapunto ante los diagnósticos y soluciones oficiales, introduciendo desde la racionalidad científica análisis e interpretaciones que denuncien situaciones injustas donde quiera se produzcan (desarrollo sostenible, política económica y distribución de la riqueza, infraestructuras, comunicación, sanidad, justicia, educación, etc.). Y planteando propuestas de intervención alternativas que coadyuven a la solución de los problemas sociales. 
¿Hemos cambiado? La perspectiva de los profesores eméritos sobre el cambio educativo en la universidad

Felipe Trillo Alonso, Miguel Zabalza Beraza y María Ainoa Zabalza Cerdeiriña

\subsection{LAS POLÍTICAS UNIVERSITARIAS}

A tenor de la definición tan concisa que nos dimos sobre lo que es una reforma educativa, las aportaciones de los eméritos al respecto han sido tan fáciles de identificar como escasas, pero no por ello carentes de interés. Veámoslas:

1- Yo no creo ni en los Bolonia ni en los cambios de programas ni nada... los cambios de programa son más de lo mismo pero revuelto de distinta manera, ... por eso no creo en reformas, en las ideas de corto plazo, porque la educación, como la cultura, son procesos lentos ... Los que creen que se pueden cambiar las cosas de un día para otro no saben qué es la ciencia, no saben qué es la educación, no saben qué es la cultura. (E-1)

2- Del plan Bolonia, yo creo que está muy bien la idea, ahora no queda más que ir por donde vaya ... lo que más me disgusta... los políticos, [que] no le han dado esa importancia ... [pues] ... lo que tienen que hacer es dar más dinero. (D-1)

3- Yo estaría preocupadísimo por el tema de Bolonia, de incorporar Bolonia, sobre todo el sistema de las tutorías, que es clave, no tenemos experiencia ninguna, la gente no lo sabe hacer, entonces a formar a la gente en el tema de las tutorías. (M-2)

4- Bolonia lo podría dar, si se hiciera de otra manera. Si intervinieran más los colegios profesionales, ... son los que tienen los problemas con los que se enfrenta la profesión. Yo creo que la universidad se debe encaminar primero hacia una mayor profesionalización de la formación universitaria y por otra parte, debería caminar hacia un mayor compromiso crítico con la sociedad. (Psi-1)

5- Hice propuestas de cambiar la carrera tal y como yo la veía ... Por una parte era reducir las 25 asignaturas a unas 19, porque había muchas... Un poco en la línea de Bolonia, casualmente, ... aparte de eso, daba mucha importancia a la práctica, a la práctica jurídica ... también a evitar el dogmatismo y promover el debate. (D-1)

6- Lo de Bolonia es una tomadura de pelo porque no se sigue Bolonia más que con el nombre. El postgrado de Bolonia supone un cambio en la enseñanza, que sea personalizada, que sea tutorizada, aquí nada, tú vas a Derecho y te encuentras con los 200 alumnos de toda la vida alli sentados y aquí no ha pasado nada, ¿no? (Fia -1)

Todas las aportaciones de los eméritos se refieren al denominado "Plan Bolonia". Sorprende la ausencia de menciones a leyes de reforma 
educativa como la LRU y la LOU que, al menos formalmente transformaron la universidad española. También sorprende que, sobre el proceso de convergencia al Espacio Europeo de Educación Superior (EEES), las respuestas se hayan quedado en aspectos superficiales: todo se reduce, en el mejor de los casos, a un cambio de programas y de metodologías para los que hace falta financiación. Lejos de los grandes objetivos políticos del espacio común europeo, el reconocimiento entre las universidades, la Europa del conocimiento, el intercambio de estudiantes.

Es interesante el primer texto en el que se pone de manifiesto que los cambios impuestos por decreto y con urgencia, con desconocimiento y hasta desconsideración de los contextos y agentes que han de llevarlos a cabo, son difícilmente viables y, menos aún, rápidos de implementar. También el segundo con su denuncia sobre la insensibilidad de los políticos para con la educación y con su reivindicación de una mayor financiación. Bolonia a "coste cero", resulta una propuesta imposible.

Es muy interesante la tercera respuesta, pues destaca lo que estaba Ilamado a ser un cambio fundamental y que, en nuestra opinión, se ha visto frustrado hasta el momento, y no sólo por falta de formación como reclama nuestro informante sino por falta de recursos. Hablamos del sistema de tutorías y la necesidad de hacer grupos de aula mucho más pequeños para que esa voluntad de llevar a cabo una orientación del aprendizaje contingente a las necesidades de los estudiantes se hiciera efectiva.

La cuarta y quinta respuestas abordan también aspectos claves: la necesaria atención a la profesionalización que no es lo mismo que la empleabilidad, la reducción de materias (nuestro informante está pensando en la sobresaturación de contenido) y la ampliación de las prácticas. Todos ellos cambios que, sin duda, se han acometido, si bien con irregular fortuna. La última manifestación recogida nos lleva a cavilar sobre un cierto engaño o mentira y a recodar aquello de Ortega y Gasset (1930) acerca de que se puede aspirar a ser lo que se quiera ser, pero no se puede fingir ser lo que no se es.

\section{Universidad y profesorado}

Son muchos y variados los aspectos a considerar aquí. Las consideraciones sobre el profesorado y la enseñanza constituyen dos elementos clave de la idea de universidad. Es un apartado en el que la mirada de los eméritos es capaz de encontrar luces y sombras. 
¿Hemos cambiado? La perspectiva de los profesores eméritos sobre el cambio educativo en la universidad

Felipe Trillo Alonso, Miguel Zabalza Beraza y María Ainoa Zabalza Cerdeiriña

Los eméritos ponen en un lugar central de la acción universitaria a los docentes. Ellos y ellas son su corazón, su fuerza, su masa crítica. Estas son sus respuestas:

1- La universidad, para su supervivencia, tiene que ir a la búsqueda de maestros más que de profesores. (Q-2)

2- Devolver al profesor esa seguridad de que es alguien importante en la sociedad, no un "trabajador de la enseñanza", sin más, con fraseología sindical. (C-1)

3- Que el profesor sienta que se valora su dedicación a la docencia (y no solo mediante encuestas anónimas a los estudiantes, más o menos acertadas). (G-1)

4- Lo mismo que para la investigación hay que desarrollar unas competencias, hay que hacerlo para la docencia, son necesarias; no le otorgan el título de investigador a cualquiera, pero sí el de docente (sin necesidad de haber desarrollado las competencias previas). (P-1)

5- Hoy por hoy, la tarea docente está minusvalorada frente a la labor investigadora por motivos de promoción interna del profesorado ... el factor condicionante para la obtención de la plaza objeto de concurso reside en presentar una buena y objetivable labor investigadora, frente a las características pedagógicas que conlleva la misión suprema de la universidad, que es la enseñanza. (Q-2)

6- En algunos ámbitos médicos, como farmacología, muy experimentales, se pasó al polo contrario, al de que la docencia no sirve para nada y sólo vale la investigación ... se ha creado este ídolo de las publicaciones de impacto. (M-1)

7- La investigación es fundamental: los profesores que no investigan no creo que puedan saber nunca mucho de lo que hacen. Pero cuidado con el exceso: en algunas disciplinas la investigación es la única cosa importante, aunque seas un profesor horrible, y eso no puede ser ... Por ejemplo, si se permite que investigadores Ramón y Cajal o Parga Pondal se estabilicen por la vía docente, cuando son investigadores y muchos no saben qué hacer delante de un alumno. Creo que eso es un grave daño. (M-2)

8- El esquema que tradicionalmente se ha barajado, incluso para la evaluación de profesores es: 60 investigación, 30 docencia, 10 proyección. Pero no estoy de acuerdo: lo primero es la docencia, ahí es donde habría que dedicar más tiempo y tener el reconocimiento en la evaluación con mayor peso. La investigación son habilidades distintas no necesariamente vinculadas al hecho de ser buen profesor. (P-1) 
Felipe Trillo Alonso, Miguel Zabalza Beraza y María Ainoa Zabalza Cerdeiriña

9- veo un cierto desánimo en las generaciones jóvenes básicamente porque no se les ha diseñado una hoja de ruta ... hay gente con muy buena preparación que tienen unas becas precarias y desde luego en absoluto competitivas ... la universidad en este momento tiene una urgencia máxima de definir una carrera universitaria donde la gente sepa que puede seguir ese camino y que no queda digamos al criterio de una comisión evaluadora de los programas tipo Ramón y Cajal ... además, así se seleccionan personas que son ajenas a las necesidades de los departamentos, y nadie mejor que un departamento para conocer cuáles son sus necesidades, sin riesgo de incurrir por ello en nepotismo. (M-1)

10- ahora estamos viviendo una crisis terrible ...Porque hay cada vez menos profesores, en mi facultad, vamos a ver, yo ahora me jubilo y no me van a substituir que yo sepa por nadie, se murió una persona de historia y no la substituyeron, otra se jubiló y no la substituyeron, otras dos más se jubilaron y no las substituyeron. O sea, se está en una política de gasto mínimo y sería para escribir largo y tendido. (Fia -1)

Del análisis del conjunto de las manifestaciones recogidas se desprende que la función docente es un tema clave para los profesores eméritos. Probablemente ellos y ellas son eméritos por sus méritos en investigación, pero sus respuestas muestran una gran consideración por la enseñanza. Pese a su brevedad y concisión, las tres primeras manifestaciones son una llamada de atención para mantener o recuperar criterios como el reconocimiento, respeto y estima por la función docente. La apelación a la figura del maestro -que forma- en contraste con la del profesor -que informa-, resulta muy elocuente.

En efecto, las cinco manifestaciones siguientes plantean sin ambages esa confrontación entre la docencia y la investigación. Por eso mismo, aunque todos sostienen la necesidad de realizar ambas tareas, también advierten y hasta condenan la minusvaloración de la docencia.

Justamente, para evitarla, en la novena manifestación encontramos una reclamación sobre la obligación, casi moral, de definir una carrera docente. El análisis intra-sujeto de este profesor nos ayuda a entender su queja: no puede conservar a jóvenes que se han formado en su departamento y eso le duele. Más allá de los riesgos del nepotismo y la endogamia resulta frustrante que todo el esfuerzo formativo se quede en nada. También lo es que la falta de expectativas laborales vaya minando la fe y capacidad de esfuerzo de nuestros becarios e investigadores. Todo ello, 
¿Hemos cambiado? La perspectiva de los profesores eméritos sobre el cambio educativo en la universidad

Felipe Trillo Alonso, Miguel Zabalza Beraza y María Ainoa Zabalza Cerdeiriña

añadido a la no reposición de las plazas está provocando una notable precarización de la enseñanza. Según esto, estamos aún lejos de prestar la atención que se merece el reconocimiento de la docencia como un indicador relevante de la calidad.

\section{La Innovación educativa}

Aunque sin cambio no hay innovación, no todos los cambios dan lugar a innovaciones relevantes. Y eso es aún más probable que suceda en aqueIlas instituciones poco propicias al cambio, como es el caso. Con todo, no somos partidarios de una visión catastrofista. En honor a la verdad, hay que reconocer que no pocas cosas han cambiado en la universidad. Algunas de ellas representan, incluso, un "cambio profundo en las concepciones de los directamente involucrados".

El acuerdo de los eméritos es casi unánime: coinciden en señalar como cambios a mejor los que a continuación presentamos.

1- la proyección de la universidad como centro de pensamiento, no es la consecuencia ni del tiempo que se asigna a esto ni de los recursos que se asignan sino de la actitud mental de los que estamos en la universidad ... uno puede dar las clases enseñando a pensar o puede dar las clases evitando que los chicos piensen. (E-1)

2- lo más importante es estimular el descubrimiento, ... provocar que tengas ganas de saber el por qué ... despertar el entusiasmo ... ayudar al estudiante reflexivo ... la responsabilidad [en esto] de un profesor es tremenda... (D-1)

3- lo importante es que los alumnos entiendan lo que estudian versus dar el programa completo de la materia. (F-1)

4- con el paso de los años te vas dando cuenta de que lo que hay que hacer es formar gente que sepa entender las cosas, aunque no se termine la última lección y el último detalle; los alumnos tiene que pensar por cuenta propia, a leer revistas, esto es lo más importante, hacerlos pensar y madurar, más que en empeñarse en dar el último detalle del programa. (Q-1)

5- Enseñar a pensar. Esto es, a analizar, sintetizar y exponer una determinada temática. La importancia de profundizar en el tronco de las materias y no en las ramas. Y enseñar a relacionar unos troncos con otros. (Q-2)

6- lo importante de la tarea docente es que el alumno se forme y que entienda la asignatura, es decir, que pueda razonar y que después pueda aplicar los conocimientos que ha adquirido a cuestiones prácticas. (Fa-1) 
7- Siempre he creído que es más importante que aprendan a aprender que el hecho de acumular conocimientos momentáneamente. (G-1)

8- Lograr que los alumnos lleguen a ser capaces de hacer síntesis personales de los temas de estudio. Que sean capaces de asumir retos, que no se enganchen a lo ya aprendido y memorizado. (BA-1)

9- Es importante que aprendan unos conocimientos, pero no todos los conocimientos: hay profesores que se preocupan por dar hasta la última lección del programa; para mí eso no es lo importante, sino que los estudiantes maduren y aprendan matemáticas, pero al mismo tiempo aprendan a trabajar con colegas suyos en proyectos que les damos. (IdM-1)

Los profesores eméritos -quizás por eso lo son-, rechazan el enciclopedismo huero y la memorización estéril. En sus manifestaciones apuestan decididamente por desarrollar las habilidades de pensamiento de los estudiantes, entendidas no solo como la posibilidad de elucubrar, sino como un atreverse a cuestionar todos los supuestos mediante un riguroso ejercicio de reconstrucción crítica de lo ya sabido y de puesta a prueba de nuevas hipótesis alternativas (Trillo, Zabalza-Cerdeiriña y Parada, 2015).

Según esto, nuestros informantes incorporan aquí dos de las propuestas nucleares de la pedagogía universitaria actual: la enseñanza centrada en el Estudiante y la promoción de un aprendizaje significativo y autónomo. Al que la última respuesta añade la cualidad de cooperativo.

\section{Conclusiones}

Las conclusiones elaboradas a partir de las respuestas de los eméritos se han ido diseminando al hilo de los comentarios sobre las mismas. Llegados a este punto quisiéramos anotar, tan solo, algunas reflexiones finales:

1. El sentido subjetivo del posicionamiento de cada profesor/a emérito. Subjetivo en un doble sentido; (a) porque refleja su opinión o valoración personal; (b) porque las ideas que expresan las han subjetivado, las han hecho suyas. Hablan de la universidad como algo que les pertenece y a la que pertenecen, como algo suyo, personal.

2. La naturaleza de la universidad y su dinámica alberga dilemas que parecen irresolubles. La variedad de lecturas sobre lo que la insti- 
¿Hemos cambiado? La perspectiva de los profesores eméritos sobre el cambio educativo en la universidad

Felipe Trillo Alonso, Miguel Zabalza Beraza y María Ainoa Zabalza Cerdeiriña

tución debiera ser y hacer se ha hecho estructural: docencia vs. investigación; orientación profesionalizante vs. cultural; vinculación a las demandas sociales vs. autonomía y distancia; aprendizajes operativos vs. valores y capacidad crítica. Como en todo dilema, la única solución es tratar de buscar el equilibrio entre los polos contrapuestos.

3. Se ha podido comprobar el notable impacto que ha tenido Bolonia. Incluso siendo una reforma frustrada en sus elementos esenciales su presencia en la agenda universitaria ha sido muy potente y persuasiva.

4. La priorización del buen aprendizaje sobre la cantidad de aprendizajes. El que estas ideas las hayan repetido prácticamente todos los eméritos hace pensar que esa creencia depende mucho de la evolución personal de los docentes, como si fuera una de las últimas fases del crecimiento profesional de los mejores profesores.

\section{Referencias bibliográficas}

Bain, K. (2006). Lo que hacen los mejores profesores universitarios. Valencia: Servei de publicacións, Universitat de Valencia.

Barnett, R. (1992). Improving Higher Education. Total Quality Care. London: Society for Research into Higher Education and Open University.

Barnett, R. (1997). Higher Education: a critical business. London: Society for Research into Higher Education and Open University.

Barnett, R. and Di Napoli, R. (2007). Changing Identities in Higher Education. London: Routledge.

Becerra-Fernández, I. y Sabherwal, R. (2010). Knowledge management: systems and processes. New York: Sharpe

Bolívar, A. (2002). Cómo mejorar los centros educativos. Madrid: Síntesis.

Bolívar, A. (2006). La identidad profesional del profesorado de secundaria: crisis y reconstrucción. Malaga: Aljibe

Bolívar, A. y Rodríguez, J.L. (2002). Reformas y Retórica: La reforma Educativa de la LOGSE. Málaga: Aljibe.

Díaz Barriga, (2010). Los profesores ante las innovaciones curriculares. Revista iberoamericana de educación superior,1(1), 37-57.

Dye, J.F.; Schartz, I.M.; Rosenberg, B.A. \& Coleman, S.T. (2000). Constant Comparison Method: a Kaleidoscope of Data. The Qualitative Report, 4(1), 1-10.

Escudero Muñoz, J.M. (1999). El cambio en educación, las reformas y la renovación pedagógica. En J.M. Escudero Muñoz (Coord.). Diseño, desarrollo e innovación del currículum, pp. 67-98. Madrid: Síntesis. 
¿Hemos cambiado? La perspectiva de los profesores eméritos sobre el cambio educativo en la universidad

Felipe Trillo Alonso, Miguel Zabalza Beraza y María Ainoa Zabalza Cerdeiriña

Escudero Muñoz, J.M. (2002). La Reforma de la Reforma. ¿Qué calidad, para quiénes? Barcelona: Ariel.

Escudero Muñoz, J.M. y González, M.T. (1987). Innovación educativa. Teorías y procesos de desarrollo. Barcelona: Ed. Humanitas.

Feixas, M. (2002). Los cambios en la docencia del profesor universitario. Tesis doctoral. Dpto. de Pedagogía Aplicada. Universidad Autónoma de Cataluña.

Finkel, D. (2008). Dar clase con la boca cerrada. Valencia. Servicio de publicaciones Univ. de Valencia.

Fox, D. (1983). Personal Theories of Teaching. Studies in Higher Education, 8, 151-163.

Fullan, M. (1982). The Meaning of Educational Change. New York: Teacher College Press.

Fullan, M. (2002). Los nuevos significados del cambio en la educación. Barcelona: Octaedro.

Gairin, J. ( 2010). Innovación y cambio en las instituciones educativas. En A. Medina y M. Sevillano (Coords.). Diseño, desarrollo e innovación del currículum, pp. 49-122. Madrid: Universitas.

Glaser, B. \& Strauss, A. (1967). The Discovery of Grounded Theory: strategies of qualitative research. London: Wiedenfeld and Nicholson.

Gow, L. \& Kember, D. (1993). Conceptions of Teaching and Their Relationship to Student Learning. British Journal or Educational Psychology, 63, 20-33.

Guarro, A. (2005). Los procesos de cambio educativo en una sociedad compleja. Madrid: Pirámide.

Hativa, N. \& Goodyear, P. (2002) (Eds.). Teacher thinking, beliefs and knowledge in higher education. Dordrecht, The Netherlands : Kluwer Academic Publishers.

Hankel, M. (2000). Academic Identities and Policy Change in Higher Education. London: Jessica Kingsley

Kember, D. (2001). Beliefs about Knowledge and the process of Teaching and Learning as a Factor in Adjusting to Study in Higher Education. Studies in Higher Education, 26 (2), 205-221.

Koppelman, H. (2005). Capturing pedagogical knowledge. Recent Research Developments in Learning Technologies (2005). www.formatex.org/200g

Moreno, A (2002). Historia-de-vida e Investigación. Caracas: Cip.

Ortega y Gasset, J. (1930). Misión de la Universidad: sobre reforma universitaria. Madrid: Revista de Occidente.

Rivas, M. (2000). Innovación educativa: teoría, procesos y estrategias. Madrid: Síntesis.

Samuelowicz, K. (1999). Academics' Educational Beliefs and Teaching Practices. Thesis. Griffith University. Australia.

Samuelowicz, K. \& Bain, J.D. (2001). Revisiting Academics' Beliefs about Teaching and Learning. Higher Education, 41(3), 299-325.

Stanley, D.J. \& Trinkle, D.A. (2011). The changing landscape of Higher Education. Educause Review, 46, 15-32.

Swanger, D. (2016). Innovation in Higher Education. En www.fmcc.edu/about/files/2016/06/Innovation-in-Higher-Education.pdf

Trillo, F. , Zabalza-Cerdeiriña, Ma . A. y Parada Gañete, A. (2015): La visión del 
¿Hemos cambiado? La perspectiva de los profesores eméritos sobre el cambio educativo en la universidad

Felipe Trillo Alonso, Miguel Zabalza Beraza y María Ainoa Zabalza Cerdeiriña

profesorado emérito sobre los estudiantes: aprendiendo de los mayores. REDU, 13, 2, 143-169.

Zabalza Beraza, M.A. y Zabalza-Cerdeiriña, M.A . (2012). Innovación y cambio en las instituciones educativas. Rosario, Argentina: Homo Sapiens. 